\title{
Attracting and Natural Invariant Varieties for Polynomial Vector Fields and Control Systems
}

\author{
Niclas Kruff ${ }^{1} \cdot$ Christian Schilli $^{2} \cdot$ Sebastian Walcher ${ }^{1}$ (D) Eva Zerz ${ }^{2}$ \\ Received: 11 January 2019 / Accepted: 23 October 2019 / Published online: 30 January 2020 \\ (c) The Author(s) 2020
}

\begin{abstract}
We discuss real and complex polynomial vector fields and polynomially nonlinear, input-affine control systems, with a focus on invariant algebraic varieties. For a given real variety we consider the construction of polynomial ordinary differential equations $\dot{x}=f(x)$ such that the variety is invariant and locally attracting, and show that such a construction is possible for any compact connected component of a smooth variety satisfying a weak additional condition. Moreover we introduce and study natural controlled invariant varieties (NCIV) with respect to a given input matrix $g$, i.e. varieties which are controlled invariant sets of $\dot{x}=f(x)+g(x) u$ for any choice of the drift vector $f$. We use basic tools from commutative algebra and algebraic geometry in order to characterize NCIV's, and we present a constructive method to decide whether a variety is a NCIV with respect to an input matrix. The results and the algorithmic approach are illustrated by examples.
\end{abstract}

Keywords Invariant set $\cdot$ Attractor $\cdot$ State feedback $\cdot$ Lyapunov function

Mathematics Subject Classification 93B52 $\cdot 34 \mathrm{C} 45 \cdot 93 \mathrm{C} 10 \cdot 37 \mathrm{C} 10$

\section{Introduction}

In the present paper, we discuss ordinary differential equations with polynomial right hand side

$$
\dot{x}=f(x)
$$

\footnotetext{
Sebastian Walcher

walcher@matha.rwth-aachen.de

1 Lehrstuhl A für Mathematik, RWTH Aachen, 52056 Aachen, Germany

2 Lehrstuhl D für Mathematik, RWTH Aachen, 52056 Aachen, Germany
}

Birkhäuser 
as well as polynomial control systems

$$
\dot{x}=f(x)+g(x) u
$$

over $\mathbb{K}^{n}$ (with $\mathbb{K}=\mathbb{R}$ or $\mathbb{C}$ ), with $f$ a $\mathbb{K}^{n}$-valued polynomial, $g$ a polynomial $n \times m$ matrix, and $u$ having values in $\mathbb{K}^{m}$. Considering complex systems will turn out to be useful also for the real case. We are mainly interested in polynomial state feedbacks $\alpha: \mathbb{K}^{n} \rightarrow \mathbb{K}^{m}$ and the corresponding closed loop system

$$
\dot{x}=f(x)+g(x) \alpha(x) \text {. }
$$

Overall we are concerned with invariant algebraic varieties of polynomial vector fields, and with the construction of polynomial vector fields that leave a given variety invariant. This approach has proven quite fruitful in qualitative investigations and integrability problems; see e.g. [6,7], Llibre et al. [13] and the monograph by Zhang [22]. For control systems, the notion of controlled invariant variety $(C I V)$ was introduced and investigated in [21]. One calls the algebraic variety $V$ controlled invariant for system (2) if there exists a polynomial state feedback $\alpha$ such that $V$ is an invariant set for (3). This extends the notion of controlled invariant subspaces for linear systems which was introduced by Basile and Marro in 1969. The geometric approach for linear systems provides the theoretical basis for solving certain decoupling and noninteracting problems; see [2] for a comprehensive survey. The theory has been generalized to nonlinear systems by Isidori [10] and several other authors. Recently, some progress has been made in the area of nonlinear polynomial systems; see [15-17,21], and Yuno and Ohtsuka [19,20], where it is shown that methods from symbolic computation can be used to test the conditions for controlled invariance of varieties constructively.

The purpose of the present work, which is in part based on the doctoral dissertations $[11,17]$ by two authors of the present manuscript, is twofold. First, for real systems (1) there is interest not only in invariance of algebraic varieties but also in attractiveness. For the "inverse problem" to find suitable vector fields for a given variety, we provide some general constructions of vector fields with prescribed Lyapunov functions. Our main result here is concerned with a compact connected component of a smooth variety: Given one (technical) additional condition, we prove the existence of a polynomial vector field for which this component is locally exponentially attracting. Second, for control systems we introduce the notion of natural controlled invariant variety $(N C I V)$ with respect to a given input matrix $g$, by requiring that this variety can be made invariant for (3) with arbitrary drift $f$ by a suitable choice of the feedback $\alpha$. For real systems we will also introduce a slightly weaker form $(R N C I V)$ by allowing for rational feedbacks whose denominators have no real zeros. One motivation for introducing these notions is the observation that they ensure robustness with respect to perturbations of the drift. Moreover, from a computational point of view, there are cases where it is possible to construct NCIV's for a given $g$, whereas no solution method is currently known for the general problem of constructing CIV's for given $f$ and $g$. Our main result (Theorem 2) provides a precise characterization of NCIV's and RNCIV's, and allows an algorithmic verification of the relevant properties. We illustrate all the results by (classes of) examples. 


\section{Preliminaries}

We first introduce some notation, and note basic facts from commutative algebra and algebraic geometry that may be found in Kunz [12] and Shafarevich [18], for instance. Moreover we recall some familiar notions related to ordinary differential equations.

\subsection{Notation}

We abbreviate $R=\mathbb{K}\left[x_{1}, \ldots, x_{n}\right]=\mathbb{K}[\mathbf{x}]$. For a subset $S \subseteq R$, we write $\mathcal{V}(S)$ for the set of common zeros (in $\mathbb{K}^{n}$ ) of all elements of $S$; the set $\mathcal{V}(S)$ is then called an algebraic variety. Given an algebraic variety $V \subseteq \mathbb{K}^{n}$, one has the ideal $\mathcal{J}(V)$ of all polynomials vanishing on $V$. Given a generator set $\varphi_{1}, \ldots, \varphi_{k}$, we will fix the notation

$$
\mathcal{I}:=\mathcal{J}(V)=\left\langle\varphi_{1}, \ldots, \varphi_{k}\right\rangle
$$

throughout the paper. By $J \in R^{k \times n}$ we denote the Jacobian of $\left(\varphi_{1}, \ldots, \varphi_{k}\right)$, thus

$$
J=\left(J_{i \ell}\right) \text { with } J_{i \ell}=\partial_{\ell} \varphi_{i}
$$

The dimension $\operatorname{dim}(V)$ of $V$ is defined as the Krull dimension of $R / \mathcal{J}(V)$. The variety $V$ is a finite union of irreducible components. If all the irreducible components have the same dimension, $V$ is called equidimensional. The tangent space at $a \in V$ is given by

$$
T_{a} V=\operatorname{ker}_{\mathbb{K}}(J(a)), \quad \text { with } \operatorname{dim}\left(T_{a} V\right)=n-\operatorname{rank}(J(a)) .
$$

A point $a \in V$ is called simple (or regular, or nonsingular) if $\operatorname{dim}\left(T_{a}(V)\right.$ ) is equal to the dimension of the (unique) irreducible component of $V$ that contains $a$. Finally, the variety $V$ is said to be smooth if all $a \in V$ are simple points.

Given the ordinary differential equation (1), we call a subset $M$ of $\mathbb{K}^{n}$ invariant (resp. positively invariant, resp. negatively invariant) if for any $y \in M$ the solution orbit (resp. the positive semi-orbit, resp. the negative semi-orbit) through $y$ is contained in $M$. We call a closed invariant set $M$ (locally) attracting for (1) if there is a neighborhood $U$ of $M$ such that for any $y \in U$ the solution $z(t)$ with initial value $y$ exists for all $t \geq 0$ and satisfies dist $(z(t), M) \rightarrow 0$ as $t \rightarrow \infty$ (see e.g. Perko [14], Ch. 3.2, Def. 2).

Given some (polynomial) function $\psi$, the Lie derivative $L_{f}(\psi)$ of $\psi$ with respect to a vector field $f$ is defined by

$$
L_{f}(\psi)(x)=D \psi(x) f(x)=\langle\nabla \psi(x), f(x)\rangle
$$

with $D \psi(x)=\left(\partial_{1} \psi(x), \ldots, \partial_{n} \psi(x)\right)$, and $\nabla$ denoting the gradient. 


\subsection{Some Known Results}

First we recall from [21] some definitions and a criterion for invariance of an algebraic variety with respect to a polynomial vector field. Recall that $R=\mathbb{K}\left[x_{1}, \ldots, x_{n}\right]$.

Definition 1 With the notations from above and for $i \in\{1, \ldots, k\}$ define

$$
\mathcal{N}_{i}=\operatorname{ker}_{R}\left(\partial_{1} \varphi_{i}, \ldots, \partial_{n} \varphi_{i}, \varphi_{1}, \ldots, \varphi_{k}\right)=\operatorname{ker}_{R}\left(D \varphi_{i}, \varphi_{1}, \ldots, \varphi_{k}\right) \subseteq R^{n+k}
$$

thus $\left(\psi_{1}, \ldots, \psi_{n+k}\right) \in \mathcal{N}_{i}$ if and only if $\sum_{j} \partial_{j} \phi_{i} \psi_{j}+\sum_{\ell} \phi_{\ell} \psi_{n+\ell}=0$. Moreover set $\mathcal{M}_{i}:=\pi\left(\mathcal{N}_{i}\right)$, where $\pi$ denotes the projection onto the first $n$ components. Finally let

$$
\mathcal{M}:=\mathcal{M}_{\mathcal{I}}:=\bigcap_{i=1}^{k} \mathcal{M}_{i} \subseteq R^{n} .
$$

With these notions one has algebraic characterizations of invariant varieties for Eq. (1).

\section{Lemma 1}

$$
\begin{gathered}
V \text { is invariant for } f \Longleftrightarrow L_{f}\left(\varphi_{i}\right) \in \mathcal{I} \text { for all } i=1, \ldots, k \\
\Longleftrightarrow L_{f}(\mathcal{I}) \subseteq \mathcal{I} \Longleftrightarrow J \cdot f \in \mathcal{I} \cdot R^{k} \Longleftrightarrow f \in \mathcal{M} .
\end{gathered}
$$

From the above one sees that the complexification of a real variety that is invariant with respect to a real vector field remains invariant with respect to the complexified vector field.

There are two aspects in the discussion of invariant varieties of polynomial vector fields: In the direct problem one asks for invariant varieties of a given polynomial vector field, while in the inverse problem one considers the existence and construction of polynomial vector fields that admit a given variety as invariant set. For the (easier) inverse problem one knows some general answers. The first part of the following Proposition is obvious, but the proof of the second is nontrivial.

Proposition 1 (a) Let $H$ be a polynomial vector field such that $L_{H}\left(\varphi_{i}\right)=0$ for $1 \leq i \leq m$, and $g_{1}, \ldots, g_{m}$ arbitrary vector fields. Then

$$
f=H+\sum_{i=1}^{m} g_{i} \varphi_{i}
$$

admits the invariant variety $V$.

(b) (See Bavula [3], Theorem 1.1.) If $V$ is smooth then every polynomial vector field which admits the invariant variety $V$ is of the type given in part (a).

For smooth varieties Bavula [3] also provides a precise characterization of the vector fields $H$ which admit every $\varphi_{i}$ as a first integral. For non-smooth varieties there exist further vector fields admitting $V$; see a detailed discussion of invariant curves for planar systems in [6], and also [21]. 
Remark 1 In our context, ideals without zeros are of particular relevance. From Hilbert's Nullstellensatz [12] and the real Nullstellensatz [5] one knows: An ideal $J \subseteq \mathbb{C}[\mathbf{x}]$ admits no zero if and only if it contains the constant polynomial 1 . An ideal $J \subseteq \mathbb{R}[\mathbf{x}]$ admits no zero if and only if it contains a polynomial of the form $1+s$, with $s(x) \geq 0$ for all $x$ (actually $s$ is a sum of squares of suitable rational functions).

In some situations it will be appropriate to consider rational vector fields. The pertinent facts are stated next, with the first assertion being standard (see e.g. Perko [14], Section 3.1, Remark 3). The proof of the second statement uses the fact that closures of invariant sets (with respect to the norm topology as well as the Zariski topology) are invariant.

Lemma 2 Let $\rho \in R$ and $U:=\mathbb{K}^{n} \backslash \mathcal{V}(\rho)$.

(a) Then the ordinary differential equation

$$
\dot{x}=\frac{1}{\rho(x)} f(x) \text { on } U
$$

and the restriction of (1) to $U$ have the same solution orbits, hence the same invariant sets.

(b) If no irreducible component of $V$ is contained in $\mathcal{V}(\rho)$ then $V \cap U$ is invariant for $\dot{x}=\rho(x)^{-1} f(x)$ if and only if $V$ is invariant for (1) on $\mathbb{K}^{n}$.

\section{Prescribed Attracting Invariant Varieties}

Throughout this section we assume that $\mathbb{K}=\mathbb{R}$. As before, we consider a variety $V$ with $J(V)=\left\langle\varphi_{1}, \ldots, \varphi_{k}\right\rangle$. Now the objective is to determine polynomial vector fields for which $V$, or some connected component of $V$, is invariant and locally attracting. (Global attractivity will in general be precluded for topological reasons; see Remark 4 below.)

\subsection{Lyapunov Functions}

We first construct real polynomial vector fields with certain prescribed Lyapunov functions.

Lemma 3 Let $\mathbb{K}=\mathbb{R}$.

(a) Let $z \in V$ be a simple point, and (w.l.o.g.) assume that

$$
r:=\operatorname{rank}\left(\nabla \varphi_{1} \cdots \nabla \varphi_{k}\right)(z)
$$

satisfies $r=\operatorname{rank}\left(\nabla \varphi_{1} \cdots \nabla \varphi_{r}\right)(z)$, in particular $z$ lies in an irreducible component of dimension $n-r$. Then there exist a Zariski neighborhood $U_{z}$ of $z$ and polynomial vector fields 


$$
\kappa_{z, r+1}, \ldots, \kappa_{z, n}
$$

such that

$$
\Theta_{z}:=\left(\nabla \varphi_{1} \cdots \nabla \varphi_{r} \kappa_{z, r+1} \cdots \kappa_{z, n}\right)
$$

is invertible at every point of $U_{z}$.

(b) With $z$ as above consider the matrix

$$
A_{z}:=\operatorname{adj}\left(\Theta_{z} \cdot \Theta_{z}^{t r}\right)=\operatorname{det}\left(\Theta_{z}\right)^{2} \cdot\left(\Theta_{z} \cdot \Theta_{z}^{t r}\right)^{-1}
$$

with polynomial entries. Moreover let $H_{z}$ be a vector field such that $L_{H_{z}}\left(\varphi_{i}\right)=0$ for $1 \leq i \leq r$. Then the polynomial vector field

$$
f_{z}=H_{z}-\sum_{i=1}^{r} \varphi_{i} A_{z} \nabla \varphi_{i}
$$

satisfies

$$
L_{f_{z}}\left(\varphi_{j}\right)=-\operatorname{det}\left(\Theta_{z}\right)^{2} \varphi_{j}
$$

for $1 \leq j \leq r$.

(c) Let $V_{1}:=\mathcal{V}\left(\varphi_{1}, \ldots, \varphi_{r}\right)$, and $z_{1}, \ldots, z_{\ell}$ simple points of $V_{1}$. Then the polynomial vector field

$$
f:=\sum_{j=1}^{\ell} f_{z_{j}}
$$

satisfies

$$
L_{f}\left(\varphi_{i}\right)=-\sigma \cdot \varphi_{i} ; \quad \text { with } \sigma:=\left(\sum_{j=1}^{\ell} \operatorname{det}\left(\Theta_{z_{j}}\right)^{2}\right)
$$

for $1 \leq i \leq r$. In particular, $\varphi_{i}$ is a semi-invariant for any $i \in\{1, \ldots, r\}$, hence the sets

$$
Z_{i}:=\left\{x ; \varphi_{i}(x)=0\right\}, \quad Z_{i}^{+}:=\left\{x ; \varphi_{i}(x) \geq 0\right\}, \quad Z_{i}^{-}:=\left\{x ; \varphi_{i}(x) \leq 0\right\}
$$

are invariant for $\dot{x}=f(x)$.

If rank $\left(\nabla \varphi_{1} \cdots \nabla \varphi_{r}\right)(y)=r$ for all $y \in V_{1}$ then one may choose $z_{1}, \ldots, z_{\ell}$ such that $\sigma(y)>0$ for all $y \in V_{1}$.

(d) In the setting of part (c), for any $i \in\{1, \ldots, r\}$ the restriction of $\dot{x}=f(x)$ to the invariant set $Z_{i}^{+}$admits the Lyapunov function $\varphi_{i}$, and the restriction of $\dot{x}=f(x)$ to the invariant set $Z_{i}^{-}$admits the Lyapunov function $-\varphi_{i}$. 
(e) Given the setting of part (c), let $v(t)$ be any solution of $\dot{x}=f(x)$. Then

$$
\varphi_{i}(v(t))=\varphi_{i}(v(0)) \cdot \exp \left(-\int_{0}^{t} \sigma(v(s)) d s\right)
$$

for all $t$ in the maximal interval of existence.

Proof By assumption we have

$$
\operatorname{rank}\left(\nabla \varphi_{1} \nabla \varphi_{2} \cdots \nabla \varphi_{r}\right)(z)=r
$$

hence there exist polynomial (for instance, constant) vector fields

$$
\kappa_{z, r+1}, \ldots, \kappa_{z, n}
$$

such that

$$
\nabla \varphi_{1}(z), \cdots, \nabla \varphi_{r}(z), \kappa_{z, r+1}(z), \ldots, \kappa_{z, n}(z)
$$

form a basis of $\mathbb{R}^{n}$. Therefore the matrix

$$
\Theta_{z}=\left(\nabla \varphi_{1} \nabla \varphi_{2} \cdots \nabla \varphi_{r} \kappa_{z, r+1} \cdots \kappa_{z, n}\right)
$$

is invertible at every point of an appropriate Zariski open neighborhood $U_{z}$, and part (a) is proven. As for part (b), the matrix $A_{z}$ is symmetric and positive definite on $U_{z}$ and moreover we observe that

$$
\left(\nabla \varphi_{i}\right)^{\operatorname{tr}}\left(\Theta_{z}^{-1}\right)^{\operatorname{tr}} \cdot \Theta_{z}^{-1} \nabla \varphi_{j}=\delta_{i, j}
$$

follows from

$$
\Theta_{z} \cdot e_{i}=\nabla \varphi_{i}, \quad 1 \leq i \leq r
$$

with the standard basis $e_{1}, \ldots, e_{n}$ of $\mathbb{R}^{n}$. Consequently, we get

$$
L_{f_{z}}\left(\varphi_{i}\right)=-\operatorname{det}\left(\Theta_{z}\right)^{2} \varphi_{i} \text { for all } 1 \leq i \leq r
$$

Parts (c) and (d) are then clear, while part (e) follows from the identity

$$
\frac{d}{d t} \varphi_{i}(v(t))=L_{f}\left(\varphi_{i}\right)(v(t))
$$


Remark 2 It is worth noting that the construction of $A_{z}$ in Lemma 3 ensures that the $\nabla \varphi_{i}(x)$ are orthonormal for the scalar product induced by $A_{z}(x)$ for all $x \in U_{z}$. There may be other choices which also yield this property.

In particular, for the case $r=1, \varphi_{1}=\varphi$ the construction in Lemma 3 is unnecessarily complicated, since one may choose $A$ as the identity matrix. Indeed the polynomial vector field

$$
f:=H-\varphi \cdot \nabla \varphi
$$

with $L_{H}(\varphi)=0$ satisfies

$$
L_{f}(\varphi)=-\|\nabla \varphi\|^{2} \cdot \varphi
$$

for which the conclusions of Lemma 3(d) and (e) hold, mutatis mutandis.

Lyapunov functions yield positively invariant sublevel sets (see e.g. Amann [1], Thm. 18.2) and impose restrictions on limit sets (LaSalle's principle; see e.g. Amann [1], Thm. 18.3); note also the invariance of limit sets. We obtain:

Proposition 2 Let the setting and notation of Lemma 3 be given. Then:

(a) For any $c>0$ and any $i \in\{1, \ldots, r\}$ the sets

$$
\left\{x \in Z_{i}^{+} ; \varphi_{i}(x) \leq c\right\} \text { and }\left\{x \in Z_{i}^{-} ; \varphi_{i}(x) \geq-c\right\}
$$

are positively invariant for $\dot{x}=f(x)$.

(b) Let $y \in \mathbb{R}^{n}$ such that the omega limit set $\omega(y)$ is defined and nonempty, and denote by $Y$ the largest invariant set for $\dot{x}=f(x)$ that is contained in $\mathcal{V}\left(\operatorname{det}\left(\Theta_{z_{1}}\right), \ldots, \operatorname{det}\left(\Theta_{z_{\ell}}\right)\right)$. Then

$$
\omega(y) \subseteq V_{1} \cup Y
$$

\subsection{Attracting Compact Components}

Keeping the notation from Lemma 3, we denote by $\widetilde{V}$ a connected component of $V$ which is also compact. Moreover we assume that $\operatorname{dim} \widetilde{V}=n-r$ and impose the stronger condition that

$$
\operatorname{rank}\left(\nabla \varphi_{1}, \ldots, \nabla \varphi_{r}\right)(a)=r \text { for all } a \in \widetilde{V}
$$

Remark 3 For the following we define $f$ as in Lemma 3(c), and require $\sigma(a)>0$ for all $a \in \widetilde{V}$.

By condition (7), each point $a \in \widetilde{V}$ admits a neighborhood and a diffeomorphism $\Theta$ to a neighborhood of $0 \in \mathbb{R}^{n}$ with $\varphi_{i} \circ \Theta^{-1}=x_{i}$ for $1 \leq i \leq r$. Thus locally one may assume

$$
\varphi_{1}=x_{1}, \ldots, \varphi_{r}=x_{r}
$$


Thus, there exists a compact neighborhood $K_{a}$ of $a$ such that

$$
\left(\varphi_{1}(y), \ldots, \varphi_{r}(y)\right)=(0, \ldots, 0)
$$

holds if and only if $y \in K_{a} \cap \widetilde{V}$. Since $\widetilde{V}$ is compact, there exists a compact neighborhood $N$ of $\widetilde{V}$ such that

$$
\left(\varphi_{1}(y), \ldots, \varphi_{r}(y)\right) \neq(0, \ldots, 0)
$$

for all $y \in N \backslash \tilde{V}$. Moreover we may assume that $\sigma(x)>0$ for all $x \in N$.

Theorem 1 Let $\widetilde{V}$ be a compact connected component of $V$. Then any polynomial vector field $f$ as given in Remark 3 has the following property: There exists a compact, positively invariant neighborhood $K \subseteq N$ (see Remark 3) such that $\widetilde{V} \subseteq$ int $K$ and:

(i) Each $z \in K$ satisfies $\emptyset \neq \omega(z) \subseteq \widetilde{V}$.

(ii) All solutions of $\dot{x}=f(x)$ that start in $K$ are attracted exponentially by $\tilde{V}$.

Proof Define

$$
\psi(x):=\sum_{i=1}^{r} \varphi_{i}^{2}(x) ; \quad L_{f}(\psi)(x)=-2 \sigma \psi(x)
$$

in particular $\psi$ is a Lyapunov function. Since $N$ is compact, so is its boundary $\partial N$, hence $\psi$ attains a positive minimum $c$ on $\partial N$, and the connected component $K$ of

$$
\left\{x \in \mathbb{R}^{n} ; \psi(x) \leq c\right\}
$$

which contains $\widetilde{V}$ is a compact subset of $N$. By construction, $K$ is positively invariant. From La Salle and $\sigma(x) \geq \sigma_{0}>0$ for all $x \in N$ one sees that $\omega(z) \subseteq \widetilde{V}$, and this limit set is nonempty since the positive semi-orbit is contained in the compact set $K$. Finally, with Lemma 3(e) one sees

$$
\varphi_{i}(v(t)) \leq \exp \left(-\sigma_{0} t\right) \varphi_{i}(v(0)) \text { when } v(0) \in K \cap Z_{i}^{+}, \quad 1 \leq i \leq r,
$$

and

$$
\varphi_{i}(v(t)) \geq \exp \left(-\sigma_{0} t\right) \varphi_{i}(v(0)) \text { when } v(0) \in K \cap Z_{i}^{-}, \quad 1 \leq i \leq r .
$$

In view of Remark 3, the second assertion follows.

Corollary 1 Let $\widetilde{V} \subseteq V \subseteq \mathbb{R}^{n}$ as above, in particular let (7) be satisfied; moreover let $f$ be as in Remark 3 , and $\psi=\varphi_{1}^{2}+\cdots+\varphi_{r}^{2}$ with $L_{f}(\psi)=-2 \sigma \psi$. Then, given any polynomial vector field $h$ that leaves $V$ invariant, there exists an $\varepsilon_{0}>0$ such that $\widetilde{V}$ is an attracting invariant set for

$$
f+\varepsilon h \text { as well as } \varepsilon^{-1} f+h, \quad 0<\varepsilon<\varepsilon_{0} .
$$


Proof It suffices to prove the assertion for $f+\varepsilon h$. Invariance of $V$ w.r.t. the vector field $h$ implies the existence of rational functions $\mu_{i, j}$ which are regular on $\widetilde{V}$ such that

$$
L_{h}\left(\varphi_{i}\right)=\sum_{j=1}^{r} \mu_{i, j} \varphi_{j}, \quad 1 \leq i \leq r .
$$

This follows e.g. from Theorem 4 in Shafarevich [18], Ch. II, §3, with the $\varphi_{i}$ forming a system of local parameters at every point of $\widetilde{V}$. In turn we find

$$
\begin{aligned}
L_{f+\varepsilon h}(\psi) & =-2 \sigma \psi+\varepsilon \cdot L_{h}(\psi) \\
& =-2 \sigma \psi+\varepsilon \cdot \sum_{i=1}^{r} \sum_{j=1}^{r} \mu_{i, j} \cdot \varphi_{i} \cdot \varphi_{j}
\end{aligned}
$$

Now let $N$ be as in Remark 3. Then the symmetric matrix

$$
M(x):=\operatorname{diag}(-2 \sigma(x), \ldots,-2 \sigma(x))+\varepsilon \cdot\left(\left(\mu_{i, j}(x)+\mu_{j, i}(x)\right) / 2\right)_{1 \leq i, j \leq r}
$$

is negative definite for all $x \in N$, provided that $\varepsilon$ is sufficiently small. Therefore there exists $\delta>0$ such that

$$
L_{f+\varepsilon h}(\psi)(x)<-\delta \psi^{2} \text { for all } x \in N
$$

Now the assertion follows from arguments similar to those in the proof of Theorem 1.

\subsection{Examples}

Example 1 When $r=1$, i.e. $V$ is a hypersurface, Remark 2 yields

$$
f(x)=H(x)-\varphi(x) \cdot \nabla \varphi(x)
$$

If $\widetilde{V}$ is a compact and smooth connected component then $\nabla \varphi$ does not vanish on $\widetilde{V}$, hence Theorem 1 is applicable.

- As for a concrete example, let $\varphi=x^{2}+y^{2}-1$, i.e. $\mathcal{V}(\varphi)$ is a planar circle. In this case we explicitly have

$$
f=\gamma \cdot\left(\begin{array}{c}
-2 y \\
2 x
\end{array}\right)-\left(x^{2}+y^{2}-1\right) \cdot\left(\begin{array}{l}
2 x \\
2 y
\end{array}\right)
$$

for some $\gamma \in R$. Note that $f$ admits the stationary point $(0,0)$ and therefore, $\mathcal{V}(\varphi)$ is not a globally attracting set.

- For a second example consider the variety $V=\mathcal{V}\left(y^{2}-x(x+1)(x+2)\right) \subseteq \mathbb{R}^{2}$ (an elliptic curve) which is irreducible and smooth of dimension 1 , with two connected 
components. Here we have

$$
f=\gamma \cdot\left(\begin{array}{c}
-2 y \\
-\left(3 x^{2}+6 x+2\right)
\end{array}\right)-\left(y^{2}-x(x+1)(x+2)\right) \cdot\left(\begin{array}{c}
-\left(3 x^{2}+6 x+2\right) \\
2 y
\end{array}\right)
$$

with some polynomial $\gamma$.

Example 2 Another special case is $r=n-1$, thus $\widetilde{V}$ is a compact connected component of a curve. In this situation with Lemma 3 we obtain the vector field

$$
\dot{x}=f(x):=H(x)-\sum_{i=1}^{n-1} \varphi_{i}(x) A(x) \nabla \varphi_{i}(x)
$$

and $A$ can be constructed in the following special manner: By assumption

$$
\operatorname{rank}\left(\nabla \varphi_{1} \cdots \nabla \varphi_{n-1}\right)=n-1
$$

holds on $V$. We define the vector field $\kappa$ by

$$
\kappa:=\nabla \varphi_{1} \times \cdots \times \nabla \varphi_{n-1}
$$

where $w_{1} \times \cdots \times w_{n-1}$ is the generalized cross product on $\mathbb{R}^{n}$ defined via

$$
\operatorname{det}\left(w_{1}, \cdots, w_{n-1}, w_{n}\right)=\left\langle w_{n}, w_{1} \times \cdots \times w_{n-1}\right\rangle .
$$

Then $\kappa(v)$ is orthogonal to each vector $\nabla \varphi_{i}(v)$ for any $v \in \mathbb{R}^{n}$, and furthermore $\kappa(z) \neq 0$ for all $z \in V$. Thus

$$
\Theta=\left(\nabla \varphi_{1} \cdots \nabla \varphi_{n-1} \kappa\right)
$$

is invertible on $V$, and for

$$
A:=\operatorname{det}(\Theta)^{2} \cdot\left(\Theta \cdot \Theta^{\operatorname{tr}}\right)^{-1}
$$

Theorem 1 is applicable.

Again we give a concrete example. Let $n=3$ and consider the ideal

$$
I:=\left\langle\varphi_{1}, \varphi_{2}\right\rangle \subseteq \mathbb{R}\left[x_{1}, x_{2}, x_{3}\right]
$$

with

$$
\varphi_{1}:=x_{1}^{2}+x_{2}^{2}+x_{3}^{2}-1, \quad \varphi_{2}:=x_{1} .
$$


Then $V:=\mathcal{V}(I)$ is the intersection of the sphere $\mathbb{S}^{2}$ and the plane $x_{1}=0$. Clearly, $V$ is smooth, compact and has only one connected component. Furthermore we observe

$$
\nabla \varphi_{1}=\left(\begin{array}{l}
2 x_{1} \\
2 x_{2} \\
2 x_{3}
\end{array}\right), \quad \nabla \varphi_{2}=\left(\begin{array}{l}
1 \\
0 \\
0
\end{array}\right)
$$

Taking the cross product of both vectors and dividing by two we obtain

$$
\kappa:=\left(\begin{array}{c}
0 \\
-x_{3} \\
x_{2}
\end{array}\right)
$$

and thus we have

$$
\begin{aligned}
A & =\operatorname{det}(\Theta)^{2} \cdot\left(\Theta \cdot \Theta^{\mathrm{tr}}\right)^{-1} \\
& =\left(\begin{array}{ccc}
4\left(x_{2}^{2}+x_{3}^{2}\right)^{2} & -4 x_{1} x_{2}\left(x_{2}^{2}+x_{3}^{2}\right) & -4 x_{1} x_{3}\left(x_{2}^{2}+x_{3}^{2}\right) \\
-4 x_{1} x_{2}\left(x_{2}^{2}+x_{3}^{2}\right) & 4 x_{1}^{2} x_{2}^{2}+x_{2}^{2}+4 x_{3}^{2} & x_{2} x_{3}\left(4 x_{1}^{2}-3\right) \\
-4 x_{1} x_{3}\left(x_{2}^{2}+x_{3}^{2}\right) & x_{2} x_{3}\left(4 x_{1}^{2}-3\right) & 4 x_{1}^{2} x_{3}^{2}+4 x_{2}^{2}+x_{3}^{2}
\end{array}\right) .
\end{aligned}
$$

By Theorem 1 there exists an attracting neighborhood of $V$ with respect to any vector field

$$
f=H-\left(\varphi_{1} A \nabla \varphi_{1}+\varphi_{2} A \nabla \varphi_{2}\right)
$$

Moreover with Bavula [3] one has that $H=\rho \cdot \kappa$ for some polynomial $\rho$.

Next we demonstrate that, in contrast to the above examples, it is not generally possible to construct vector fields

$$
\kappa_{r+1}, \ldots, \kappa_{n}
$$

such that

$$
\left(\nabla \varphi_{1} \cdots \nabla \varphi_{r} \kappa_{r+1} \cdots \kappa_{n}\right)
$$

is invertible on all of $\widetilde{V}$.

Example 3 We consider the smooth, compact and connected variety given by

$$
V=\mathcal{V}\left(\varphi_{1}, \varphi_{2}\right) \subseteq \mathbb{R}^{4}
$$

with $\varphi_{1}=x_{1}^{2}+x_{2}^{2}+x_{3}^{2}+x_{4}^{2}-1$ and $\varphi_{2}=x_{1}$; thus $V$ is isomorphic to the sphere $\mathbb{S}^{2}$. Due to the hedgehog theorem (see e.g. Deimling [8], Ch. I, Thm. 3.4), one cannot construct two additional vector fields

$$
\kappa_{1}, \quad \kappa_{2} \in \mathbb{R}[\mathbf{x}]^{4}
$$


such that the vectors

$$
\nabla \varphi_{1}, \quad \nabla \varphi_{2}, \quad \kappa_{1}, \quad \kappa_{2}
$$

are linearly independent at every point of $V$. Therefore, the approach from Lemma 3(a) is not feasible in this situation.

However, the problem can be resolved via Lemma 3(c) and Theorem 1. We have

$$
\nabla \varphi_{1}=\left(\begin{array}{l}
2 x_{1} \\
2 x_{2} \\
2 x_{3} \\
2 x_{4}
\end{array}\right), \quad \nabla \varphi_{2}=\left(\begin{array}{l}
1 \\
0 \\
0 \\
0
\end{array}\right)
$$

Consider the matrices $\Theta_{1}$ and $\Theta_{2}$ given by

$$
\Theta_{1}:=\left(\begin{array}{cccc}
2 x_{1} & 1 & 0 & 0 \\
2 x_{2} & 0 & -x_{3} & -x_{2} x_{4} \\
2 x_{3} & 0 & x_{2} & -x_{3} x_{4} \\
2 x_{4} & 0 & 0 & x_{2}^{2}+x_{3}^{2}
\end{array}\right), \quad \Theta_{2}:=\left(\begin{array}{cccc}
2 x_{1} & 1 & 0 & 0 \\
2 x_{2} & 0 & -x_{4} & x_{2} x_{3} \\
2 x_{3} & 0 & 0 & -x_{2}^{2}-x_{4}^{2} \\
2 x_{4} & 0 & x_{2} & x_{3} x_{4}
\end{array}\right)
$$

Then we have

$$
\begin{aligned}
& \operatorname{det}\left(\Theta_{1}\right)^{2}=4\left(x_{2}^{2}+x_{3}^{2}\right)^{2}\left(x_{2}^{2}+x_{3}^{2}+x_{4}^{2}\right)^{2} \\
& \operatorname{det}\left(\Theta_{2}\right)^{2}=4\left(x_{2}^{2}+x_{4}^{2}\right)^{2}\left(x_{2}^{2}+x_{3}^{2}+x_{4}^{2}\right)^{2}
\end{aligned}
$$

and

$$
\sigma:=\operatorname{det}\left(\Theta_{1}\right)^{2}+\operatorname{det}\left(\Theta_{2}\right)^{2}=4\left(x_{2}^{2}+x_{3}^{2}+x_{4}^{2}\right)^{2}\left[\left(x_{2}^{2}+x_{3}^{2}\right)^{2}+\left(x_{2}^{2}+x_{4}^{2}\right)^{2}\right]
$$

vanishes if and only if $x_{2}=x_{3}=x_{4}=0$ holds. Consequently, $\sigma$ does not vanish on $V$ and Theorem 1 is applicable.

Finally we note that global attractivity of every connected component of an invariant variety is generally impossible, for topological reasons.

Remark 4 As in Example 1 consider the variety $V=\mathcal{V}\left(y^{2}-x(x+1)(x+2)\right)$ with two connected components of dimension 1, one bounded and one unbounded. Assume that $h$ is a polynomial (or smooth) vector field on $\mathbb{R}^{2}$ such that for each initial value $y \in \mathbb{R}^{2}$ the solution $z(t)$ exists on $[0, \infty)$ and satisfies dist $(z(t), M) \rightarrow 0$ as $t \rightarrow \infty$. Then there are points in $\mathbb{R}^{2} \backslash V$ which are attracted by the bounded connected component, as well as points which are attracted by the unbounded component. For each connected component its domain of attraction is nonempty and open, and $\mathbb{R}^{2}$ cannot be the union of these disjoint domains, by connectedness. Thus we obtain a contradiction. 


\section{Natural Controlled Invariant Varieties}

We now turn to control problems (2), (3), and to varieties $V$ that are invariant for (3) with appropriate feedback. Geometrically speaking, in a control problem one needs to modify $f$ via $g$ such that every solution orbit that has one point in common with $V$ is completely contained in it.

\subsection{Definition and Some Properties}

We recall and extend the definition of controlled invariance from [15].

Definition 2 (a) A variety $V$ is called controlled invariant for (2) if there exists a polynomial state feedback $\alpha \in R^{m}$ such that $V$ is invariant for the closed loop system (3).

(b) A variety $V$ is called rationally controlled invariant for (2) if there exists a rational state feedback $\alpha$ whose denominator has no zero in $\mathbb{K}^{n}$ such that $V$ is invariant for the closed loop system (3).

By Remark 1 both notions coincide for $\mathbb{K}=\mathbb{C}$, but there is a significant difference in the real case.

Now, given a polynomial input matrix $g \in R^{n \times m}$, we wish to characterize varieties which are controlled invariant for (2) for every vector field $f \in R^{n}$.

Definition 3 (a) Given a polynomial matrix $g \in R^{n \times m}$ and a variety $V$, we call $V$ rationally natural controlled invariant with respect to $g$ (briefly $R N C I V$ ) if for all $f \in R^{n}$ there exist $\alpha=\alpha_{f} \in R^{m}$ and $\psi=\psi_{f} \in R$ with $\mathcal{V}\left(\psi_{f}\right)=\emptyset$ such that $V$ is invariant for $\dot{x}=\left(\psi_{f} \cdot f+g \cdot \alpha_{f}\right)(x)$.

(b) If $\psi_{f}=1$ can be chosen then we call $V$ natural controlled invariant with respect to $g$ (briefly NCIV).

Remark 5 (a) A necessary requirement for the RNCIV property of a smooth variety $V$ is the "pointwise condition"

$$
T_{a}(V)+\mathbb{K} g_{1}(a)+\cdots+\mathbb{K} g_{m}(a)=\mathbb{K}^{n} \quad \text { for all } a \in V
$$

Indeed, if this condition fails at the point $a$ then take any polynomial drift $f$ with $f(a) \notin T_{a}(V)+\operatorname{im}_{\mathbb{K}} g(a)$. Obviously there exists no rational (or smooth) feedback $\alpha$ such that $f+g \alpha$ leaves $V$ invariant.

(b) The requirement of natural controlled invariance ensures robustness with respect to perturbations of the drift, in a dual sense:

(i) Whenever the drift is altered from $f$ to $\tilde{f}$ then there exist $\psi_{\tilde{f}}$ and $\alpha \tilde{f}$ such that $V$ is invariant for $\dot{x}=\left(\psi_{\tilde{f}} \cdot \tilde{f}+g \cdot \alpha \tilde{f}\right)(x)$. This holds by definition.

(ii) Let a (smooth) vector field $f_{1}$ be given. Then for sufficiently small $\varepsilon$, every nonstationary point $a \in V$ of $f+\psi^{-1} g \alpha$ admits a neighborhood $U$ and a local invariant manifold $V^{*}$ for the perturbed system

$$
f+\varepsilon f_{1}+\psi^{-1} g \alpha
$$


with $V^{*}$ close to $V$ (with respect to the Hausdorff distance). This is a direct consequence of standard dependency theorems.

While for every given variety it is easy to construct nontrivial polynomial vector fields that leave this variety invariant (see Proposition 1), there exist controls which do not admit interesting rationally natural controlled invariant varieties. We look at a simple example.

Example 4 (a) In $\mathbb{R}^{2}$ the input matrix

$$
g(x)=\left(\begin{array}{c}
-x_{2} \\
x_{1}
\end{array}\right)
$$

admits no smooth RNCIV of dimension one. To see this, assume that $V=\mathcal{V}(\varphi)$ is a RNCIV, and let $y \in V$ be a point of minimal distance to 0 . Setting $\rho(x):=$ $\left(x_{1}^{2}+x_{2}^{2}\right) / 2$, the Lagrange multiplier theorem implies that either $\nabla \varphi(y)=0$ (which is impossible by smoothness of $V$ ) or

$$
\lambda \cdot \nabla \varphi(y)=\nabla \rho(y)=y \text { for some } \lambda .
$$

When $\lambda=0$ then $y=0$, and $g(y)=0 \in T_{0}(V)$; a contradiction to Remark 5(a). But when $\lambda \neq 0$ then $\langle g(y), \nabla \varphi(y)\rangle=0$ which also implies $g(y) \in T_{y}(V)$; again we obtain a contradiction.

(b) Note: It is unproblematic to generalize this example to $\mathbb{R}^{n}$, with the columns of $g$ generating the submodule of $R^{n}$ which leaves $\mathbb{S}^{n-1}$ invariant. Moreover, one has the same result for closed submanifolds (rather than varieties).

(c) Nonexistence of RNCIV's may imply scarcity of CIV's for a given drift. To see this, we continue part (a) with the particular drift $f(x)=x$. Let $V$ be a one dimensional smooth invariant variety for some $f+g \alpha$, with $y \in V$ having minimal distance to 0 . The arguments from part (a) show that $\lambda=0$, with $(f+g \alpha)(y)=0$, or

$$
0=\langle(f+g \alpha)(y), y\rangle=\langle y, y\rangle .
$$

Since $f(z)$ and $g(z)$ are linearly independent for all $z \neq 0$, we find $y=0$ in both cases. To summarize, if there exists a CIV for some $f+g \alpha$ then this variety must contain 0 . There obviously exist invariant varieties for $f=f+g \cdot 0(\alpha=0$; control is switched off), viz. all straight lines through the origin. For $\alpha(0) \neq 0$ the stationary point 0 is a focus of $f+g \alpha$, which admits no local analytic invariant curves in $\mathbb{R}^{2}$, and a fortiori no algebraic invariant curves. In case $\alpha(0)=0$ the vector field is locally analytically equivalent to $f$ by Poincaré-Dulac normal form theory (see e.g. Bibikov [4]). Conclusion At most, the input matrix $g$ can deform existing invariant curves of $f$.

\subsection{Characterization}

Our main result on natural controlled invariant varieties provides equivalent conditions for a variety $V$ to satisfy the "pointwise conditions" from Remark 5 . This can be 
used for the algorithmic verification of the NCIV property, and it is also helpful for the construction of varieties, and for the computation of feedbacks. We are mainly interested in the real case, which is the underlying reason for the introduction of rational feedbacks. On the other hand, a proper understanding of the real case requires to consider the complex setting as well.

We keep the notation from Sect. 2.1, in particular $V$ is a variety with vanishing ideal $\mathcal{J}(V)$ generated by $\varphi_{1}, \ldots, \varphi_{k}$, and its Jacobian $J$ is defined in (5).

Theorem 2 Let $V$ be equidimensional and smooth with $\operatorname{dim}(V)=d$; furthermore let $g \in R^{n \times m}$.

(a) The following statements are equivalent for $\mathbb{K}=\mathbb{R}$ as well as $\mathbb{K}=\mathbb{C}$.

(i) $V$ is rationally natural controlled invariant with respect to $g$.

(ii) For all $f \in R^{n}$ there exists $\psi_{f} \in R$ such that $\mathcal{V}\left(\psi_{f}\right)=\emptyset$ and

$$
\psi_{f} \cdot f \in \mathcal{M}+i m_{R}(g)
$$

(iii) $\operatorname{rank}((J \cdot g)(a))=\operatorname{rank}(J(a))$ for all $a \in V$.

(iv) $T_{a} V+i m_{\mathbb{K}}(g(a))=\mathbb{K}^{n}$ for all $a \in V$.

(b) In case $\mathbb{K}=\mathbb{C},(i)-(i v)$ are equivalent to

(v) $\mathcal{M}+\operatorname{im}_{R}(g)=R^{n}$.

(vi) $V$ is natural controlled invariant with respect to $g$.

(c) If $\mathbb{K}=\mathbb{R}$ and any of (ii), (iii), (iv) holds for the complexification of $R$ or $V$ respectively, then $V$ is natural controlled invariant with respect to $g$.

Proof We first prove part (a); parts (b) and (c) are rather simple consequences. Since $V$ is smooth and equidimensional, the equality $\operatorname{rank}(J(a))=n-d$ holds for all $a \in V$.

1. For a fixed $\psi_{f} \in R$ we can find an $\alpha_{f} \in R^{m}$ such that $\psi_{f} \cdot f+g \cdot \alpha_{f} \in \mathcal{M}$ if and only if $\psi_{f} \cdot f \in \mathcal{M}+\operatorname{im}_{R}(g)$. From this and Lemma 1 we get (i) $\Leftrightarrow$ (ii).

To prove equivalence of (iii) and (iv) we use a fact from Linear Algebra (see e.g. Lemma 5.46 in [17]): For matrices $A \in \mathbb{K}^{k \times n}, B \in \mathbb{K}^{n \times m}$, one has $\operatorname{rank}(A)=$ $\operatorname{rank}(A B)$ if and only if $\operatorname{ker}_{\mathbb{K}}(A)+\operatorname{im}_{\mathbb{K}}(B)=\mathbb{K}^{n}$. The assertion follows with $\operatorname{ker}(J(a))=T_{a} V$.

2. In order to show the implication (ii) $\Rightarrow$ (iv) we first prove that

$$
\mathcal{M}(a):=\{h(a) \mid h \in \mathcal{M}\} \subseteq T_{a} V \text { for all } a \in V
$$

Thus let $a \in V$ and $h \in \mathcal{M}$ be arbitrary. Then Lemma 1 implies $J \cdot h \in \mathcal{I} \cdot R^{k}$ and thus we have $0=(J \cdot h)(a)=J(a) \cdot h(a)$. Because of $\mathcal{J}(\mathcal{V}(\mathcal{I}))=\mathcal{I}$ this yields $h(a) \in \operatorname{ker}(J(a))=T_{a} V$ as desired.

Next, we prove that statement (ii) implies $\mathbb{K}^{n}=\mathcal{M}(a)+\operatorname{im}_{\mathbb{K}}(g(a))$. Combined with (8) this will yield

$$
\mathbb{K}^{n}=\mathcal{M}(a)+\operatorname{im}_{\mathbb{K}}(g(a)) \subseteq T_{a} V+\operatorname{im}_{\mathbb{K}}(g(a)) \subseteq \mathbb{K}^{n}
$$


which shows (iv). So let $v \in \mathbb{K}^{n} \subseteq R^{n}$. By (ii) there exists $\psi_{v} \in R$ with $\mathcal{V}\left(\psi_{v}\right)=\emptyset$ and $q \in \mathcal{M}, w \in R^{m}$ such that $\psi_{v} \cdot v=q+g \cdot w$. But then for every $a \in V$ we have $\psi_{v}(a) \neq 0$ and thus

$$
v=v(a)=\left(\psi_{v}(a)\right)^{-1} \cdot(q(a)+g(a) \cdot w(a)) \in \mathcal{M}(a)+\operatorname{im}_{\mathbb{K}}(g(a))
$$

as desired.

3. As a preliminary step for the proof of "(iv) $\Rightarrow$ (ii)" we strengthen the subset relation in (8) to equality, thus we prove

$$
\mathcal{M}(a)=T_{a} V \text { for all } a \in V \text {. }
$$

To see this, partition

$$
J(x)=\left(\begin{array}{ll}
A_{11}(x) & A_{12}(x) \\
A_{21}(x) & A_{22}(x)
\end{array}\right), \quad A_{11}(x) \in R^{(n-d) \times(n-d)},
$$

and assume w.l.o.g. that $A_{11}(a)$ is invertible, hence $A_{11}(v)$ is invertible for all $v$ in a neighborhood of $a$. Since $J(v)$ has rank $n-d$ for all $v \in V$, we have

$$
J(v)\left(\begin{array}{c}
z_{1} \\
z_{2}
\end{array}\right)=0 \Leftrightarrow A_{11}(v) z_{1}+A_{12}(v) z_{2}=0,
$$

whence the columns

$$
w_{1}(x), \ldots, w_{d}(x) \text { of }\left(\begin{array}{c}
-A_{11}(x)^{-1} A_{12}(x) \\
I_{d}
\end{array}\right)
$$

are defined at $a$ and form a basis of $\operatorname{ker} J(x) \subseteq \mathbb{K}\left(x_{1}, \ldots, x_{n}\right)^{n}$. Multiplying by a common denominator $\rho$ of the $w_{i}$ with $\rho(a) \neq 0$, we get

$$
\widetilde{w}_{i}:=\rho \cdot w_{i} \in \mathcal{M}, \quad 1 \leq i \leq d,
$$

and $\widetilde{w}_{1}(a), \ldots, \widetilde{w}_{d}(a) \in \mathcal{M}(a)$ are linearly independent in $\mathbb{K}^{n}$.

4. Now we show that (iv) implies (ii). Let $q_{1}, \ldots, q_{s}$ generate the module $\mathcal{M}$, and let $f \in R^{n}$. Then for every $a \in V$ there exist polynomials

$$
\rho_{a}, \mu_{a, j}(1 \leq j \leq s), \quad v_{a, \ell}(1 \leq \ell \leq m)
$$

such that $\rho_{a}(a) \neq 0$, and

$$
\rho_{a} \cdot f=\sum_{j} \mu_{a, j} q_{j}+\sum_{\ell} v_{a, \ell} g_{\ell}
$$

To verify this, assume w.l.o.g. that

$$
q_{1}(a), \ldots, q_{d}(a), g_{1}(a), \ldots, g_{n-d}(a)
$$


span $\mathbb{K}^{n}$, and use Cramer's rule over $\mathbb{K}\left(x_{1}, \ldots, x_{n}\right)$.

Due to Hilbert's basis theorem [9, Thm. 1.3.5], the variety $V$ is quasi-compact [12, Prop. 1.3]. Therefore there exists $p \in \mathbb{N}$ and $\left\{a_{1}, \ldots, a_{p}\right\} \subseteq V$ such that the $\rho_{i}:=\rho_{a_{i}}$ have no common zero in $V$, hence $\rho_{1}, \ldots, \rho_{p}$ and $\varphi_{1}, \ldots, \varphi_{k}$ have no common zero in $\mathbb{K}^{n}$. By the Nullstellensatz (see Remark 1) there exist $\beta_{i}, \eta_{j} \in R$ such that

$$
\sum \rho_{i} \beta_{i}+\sum \varphi_{j} \eta_{j}=1+\sigma
$$

with $\sigma=0$ in case $\mathbb{K}=\mathbb{C}$, and $\sigma(x) \geq 0$ for all $x$ when $\mathbb{K}=\mathbb{R}$. Abbreviating $\mu_{i j}:=\mu_{a_{i}, j}$ and $v_{i k}:=v_{a_{i}, k}$, summation yields

$$
(1+\sigma) f=\sum_{j} \varphi_{j}\left(\eta_{j} f\right)+\sum_{i, j} \beta_{i} \mu_{i j} q_{j}+\sum_{i, \ell} \beta_{i} v_{i, \ell} g_{\ell} .
$$

The first two sums on the right hand side lie in $\mathcal{M}$, since $\mathcal{I} \cdot R^{n} \subseteq \mathcal{M}$, and the last sum lies in $\operatorname{im}_{R}(g)$.

5. For $\mathbb{K}=\mathbb{C}$ the equivalence of (ii) and (v) follows by Hilbert's Nullstellensatz (Remark 1); this shows part (b). Finally in part (c) we may choose $\psi_{f}=1$ in the complexification by (b), and then take real parts. Thus $V$ is a NCIV with respect to $g$.

\subsection{Examples}

As shown by Example 4, RNCIV's for a given $g$ do not exist in general. Moreover their construction (if existence is given) is a nontrivial task, although considerably easier than finding CIV's for given $f$ and $g$. In this section, we construct RNCIV's and NCIV's for special classes of input matrices $g \in R^{n \times m}$.

Proposition 3 For $\mathbb{K}=\mathbb{R}$, let $\psi_{1}, \ldots, \psi_{m} \in R$, and $A \in R^{n \times n}$ such that $A(z)$ is symmetric and positive semidefinite for all $z \in \mathbb{R}^{n}$. Define

$$
g(x):=A(x) \cdot\left(\nabla \psi_{1}(x), \ldots, \nabla \psi_{m}(x)\right)
$$

If $c_{1}, \ldots, c_{m} \in \mathbb{K}$ are such that

$$
W:=\mathcal{V}\left(\psi_{1}-c_{1}, \ldots, \psi_{m}-c_{m}\right)
$$

is smooth of dimension $d$, rank $\left(\nabla \psi_{1}(x), \ldots, \nabla \psi_{m}(x)\right)=n-d$ on $W$, and $A(z)$ is positive definite for all $z \in W$ then $W$ is a RNCIV for $g$, and a NCIV given the conditions of Theorem 2(c).

Proof For any $z \in W$, a positive definite scalar product $\tau_{z}$ is defined by

$$
\tau_{z}(u, v):=\langle u, A(z) v\rangle
$$


Then the vector space $\operatorname{im}_{\mathbb{K}}(g(z))$ is spanned by the $\nabla \psi_{j}(z)$, and the tangent space $T_{z}(W)$ is the $\tau_{z}$-orthogonal complement of this space. This implies the "pointwise" condition (ii) in Theorem 2(a).

Remark 6 In particular, if $A$ is given as in Lemma 3 (or $A$ is such that Remark 2 is applicable) and the conditions of Theorem 1 hold for $W$ then any drift admits a feedback such that $W$ is locally attracting for (3): First find a feedback such that $W$ is invariant for (3), then apply Corollary 1.

The next class of examples generalizes invariance properties of homogeneous polynomials, and also works in the complex setting.

Proposition 4 For an input matrix $g=\left(g_{1}, \ldots, g_{m}\right) \in R^{n \times m}$ assume there exist nonconstant $\mu_{1}, \ldots, \mu_{k} \in R$ such that

$$
L_{g_{i}}\left(\mu_{j}\right)=b_{j i} \cdot \mu_{j} \text { with some } b_{j i} \in \mathbb{K} ; \quad j \in\{1, \ldots, k\}, \quad i \in\{1, \ldots, m\} .
$$

Assume that there exist $c_{1}, \ldots, c_{k} \in \mathbb{K}$ such that

$$
Y:=\mathcal{V}\left(\mu_{1}-c_{1}, \ldots, \mu_{k}-c_{k}\right)
$$

satisfies $\mathcal{J}(Y)=\left\langle\mu_{1}-c_{1}, \ldots, \mu_{k}-c_{k}\right\rangle$, and $Y$ is equidimensional and smooth, of dimension $d$. If the matrix

$$
B=\left(b_{j i}\right) \in \mathbb{K}^{k \times m}
$$

satisfies $\operatorname{rank}(B)=n-d$ then $Y$ is a NCIV for $g$.

Proof Let $J$ be the Jacobian of $\mathcal{J}(Y)$, and $M:=\operatorname{diag}\left(\mu_{1}, \ldots, \mu_{k}\right)$. We have

$$
J \cdot g=\left(\begin{array}{c}
D \mu_{1} \\
\vdots \\
D \mu_{k}
\end{array}\right) \cdot\left(g_{1}, \ldots, g_{m}\right)=\left(\begin{array}{ccc}
b_{11} \mu_{1} & \cdots & b_{1 m} \mu_{1} \\
\vdots & & \vdots \\
b_{k 1} \mu_{k} & \cdots & b_{k m} \mu_{k}
\end{array}\right)=M \cdot B
$$

Since $\mu_{j}(a)=c_{j} \neq 0$ for all $a \in V$ and $j=1, \ldots, k$, we have $\operatorname{rank}(M(a))=k$ for all $a \in Y$ and thus

$$
\operatorname{rank}((J \cdot g)(a))=\operatorname{rank}((M \cdot B)(a))=\operatorname{rank}(M(a) \cdot B)=\operatorname{rank}(B)=\operatorname{rank}(J(a))
$$

Therefore $Y$ is smooth, and all assumptions of Theorem 2 are satisfied. The assertion follows.

Example 5 (a) Let $\mathbb{K}=\mathbb{R}$ and $n \geq 2$. Consider $g \in R^{n}$ with $g(x)=x$ and let $\mu$ be any homogeneous polynomial of degree $\ell \geq 1$. By the familiar Euler differential equation one has $L_{g}(\mu)=\ell \cdot \mu$, So, whenever $c \in \mathbb{R}$ is such that $W:=\mathcal{V}(\mu-c)$ is smooth then the assumptions of Propositions 3 and 4 are fulfilled and $W$ is a NCIV with respect to $g$.

As a special example consider the real case and $\mu=\sum_{i=1}^{n} \alpha_{i} x_{i}^{2}$, with all $\alpha_{i}>0$, and $c>0$. Here Remark 6 is applicable. 
(b) Let $\mathbb{K}=\mathbb{R}$ and

$$
g=\left(\begin{array}{ll}
g_{1} & g_{2}
\end{array}\right)=\left(\begin{array}{cc}
x_{1} & 0 \\
0 & x_{2} \\
0 & x_{3}
\end{array}\right)
$$

Then $W:=\mathcal{V}\left(x_{1}-c_{1}, x_{2}^{2}+x_{3}^{2}-c_{2}\right)$, with $c_{1} \in \mathbb{R}$ and $c_{2}>0$, is a NCIV with respect to $g$. To see this, note that the complexification of $W$ is smooth of dimension one, and for

$$
\mu_{1}=x_{1}, \quad \mu_{2}=x_{2}^{2}+x_{3}^{2}
$$

the identities

$$
L_{g_{1}}\left(\mu_{1}\right)=\mu_{1}, \quad L_{g_{2}}\left(\mu_{1}\right)=0, \quad L_{g_{1}}\left(\mu_{2}\right)=0, \quad L_{g_{2}}\left(\mu_{2}\right)=2 \mu_{2}
$$

hold; therefore

$$
B=\left(\begin{array}{ll}
1 & 0 \\
0 & 2
\end{array}\right)
$$

in the notation of Proposition 4. Consequently, we get

$$
\operatorname{rank}(B)=2=3-1
$$

and by applying Proposition 4 the assertion follows.

Example 6 We provide an application of Proposition 3. With $\mathbb{K}=\mathbb{R}$ and

$$
\psi:=x_{2}^{2}-x_{1}\left(x_{1}+1\right)\left(x_{1}+2\right)
$$

(compare Example 1), consider the input matrix

$$
g=\nabla \psi=\left(\begin{array}{c}
-\left(3 x_{1}^{2}+6 x_{1}+2\right) \\
2 x_{2}
\end{array}\right) .
$$

The elements of the module $\mathcal{M}$ are given by

$$
\rho \cdot q+\psi \cdot h
$$

with $\rho \in R$ and $h \in R^{2}$ arbitrary, and

$$
q:=\left(\begin{array}{c}
2 x_{2} \\
3 x_{1}^{2}+6 x_{1}+2
\end{array}\right)
$$


as follows e.g. from [6] (and is easily verified directly). We note that

$$
\Delta(x):=\operatorname{det}(q(x) g(x))=4 x_{2}^{2}+\left(3 x_{1}^{2}+6 x_{1}+2\right)^{2}
$$

has real zeros only at $x_{1}=(-6 \pm 2 \sqrt{3}) / 6$ and $x_{2}=0$. At these points one has $\psi(x)= \pm 2 \sqrt{3} / 9$. Thus for all $c \in \mathbb{R} \backslash\{ \pm 2 \sqrt{3} / 9\}$ Proposition 3 is applicable to $V_{c}:=\mathcal{V}(\psi-c)$. Thus for any $a \in V_{c}$ we have that $T_{a}\left(V_{c}\right)$ is spanned by $q(a)$; moreover $q(a), g(a)$ span $\mathbb{R}^{2}$.

Given any $f \in R^{2}$ we obtain

$$
\Delta(x) f(x)=\operatorname{det}(f(x), g(x)) \cdot q(x)+\operatorname{det}(q(x), f(x)) \cdot g(x)
$$

by Cramer's rule.

We now consider the special case $c=0$, for the sake of simplicity. According to step \# 4 in the proof of Theorem 2 one would determine polynomials $\beta$ and $\eta$ such that $\beta \Delta+\eta \psi=1+\sigma$, with $\sigma$ only attaining nonnegative values. But the relevant point is to find some linear combination of $\Delta$ and $\psi$ that has no real zero. In the present example, determining a Gröbner basis of $\langle\Delta, \psi\rangle$ yields one element

$$
\lambda:=\beta \Delta+\eta \psi=729 x_{2}^{8}-584 x_{2}^{6}-72 x_{2}^{4}+96 x_{2}^{2}+16,
$$

with

$$
\begin{aligned}
\eta= & 729 x_{1}^{3} x_{2}^{4}+1863 x_{1}^{2} x_{2}^{4}+729 x_{2}^{6}-108 x_{1}^{3} x_{2}^{2}+954 x_{1} x_{2}^{4}-324 x_{1}^{2} x_{2}^{2} \\
& -396 x_{2}^{4}-108 x 1^{3}-72 x_{1} x_{2}^{2}-324 x_{1}^{2}+36 x_{2}^{2}-216 x_{1} \\
\beta= & 81 x_{1}^{2} x_{2}^{4}+126 x_{1} x_{2}^{4}-12 x_{1}^{2} x_{2}^{2}-47 x_{2}^{4}-24 x_{1} x_{2}^{2}-12 x_{1}^{2}+20 x_{2}^{2}-24 x_{1}+4 .
\end{aligned}
$$

Note that $\lambda$ is a polynomial in one variable; an elementary discussion shows that $\lambda$ attains a positive minimum in $\mathbb{R}$. We thus obtain

$$
\lambda f=(\eta \psi \cdot f+\beta \operatorname{det}(f, g) \cdot q)+\beta \operatorname{det}(q, f) \cdot g,
$$

and choosing the feedback

$$
\alpha=\frac{\beta \operatorname{det}(q, f)}{\lambda}-\varepsilon^{-1} \psi
$$

(with any $\varepsilon$ ) yields a vector field which admits the invariant variety $V_{0}=V$. Choosing $\varepsilon>0$ sufficiently small yields a vector field which admits $\psi$ as a Lyapunov function in some neighborhood of the compact connected component $\widetilde{V}$ of $V$.

Acknowledgements Open Access funding provided by Projekt DEAL. N. Kruff and C. Schilli gratefully acknowledge support by the DFG Research Training Group GRK 1632 "Experimental and Constructive Algebra". 
Open Access This article is licensed under a Creative Commons Attribution 4.0 International License, which permits use, sharing, adaptation, distribution and reproduction in any medium or format, as long as you give appropriate credit to the original author(s) and the source, provide a link to the Creative Commons licence, and indicate if changes were made. The images or other third party material in this article are included in the article's Creative Commons licence, unless indicated otherwise in a credit line to the material. If material is not included in the article's Creative Commons licence and your intended use is not permitted by statutory regulation or exceeds the permitted use, you will need to obtain permission directly from the copyright holder. To view a copy of this licence, visit http://creativecommons.org/licenses/by/4.0/.

\section{References}

1. Amann, H.: Ordinary Differential Equations. De Gruyter, Boston (1990)

2. Basile, G., Marro, G.: Controlled and Conditioned Invariants in Linear System Theory. Prentice Hall, Englewood Cliffs (1992)

3. Bavula, V.V.: Generators and defining relations for the ring of differential operators on a smooth affine algebraic variety. Algebras Represent. Theory 13, 159-187 (2010)

4. Bibikov, Yu.N.: Local Theory of Nonlinear Analytic Ordinary Differential Equations. Lecture Notes in Mathematics, vol. 702. Springer, Berlin (1979)

5. Bochnak, J., Coste, M., Roy, M.-F.: Real Algebraic Geometry. Springer, Berlin (1998)

6. Christopher, C., Llibre, J., Pantazi, C., Walcher, S.: Inverse problems for invariant algebraic curves: explicit computations. Proc. R. Soc. Edinb. Sect. A. Math. 139, 287-302 (2009)

7. Christopher, C., Llibre, J., Pantazi, C., Zhang, X.: Darboux integrability and invariant algebraic curves for planar polynomial systems. J. Phys. A: Math. Theor. 10, 2457-2476 (2002)

8. Deimling, K.: Nonlinear Functional Analysis. Springer, Berlin (1985)

9. Greuel, G.M., Pfister, G.: A Singular Introduction to Commutative Algebra. Springer, Berlin (2002)

10. Isidori, A.: Nonlinear Control Systems. Springer, London (1995)

11. Kruff, N.: Local invariant sets of analytic vector fields. Ph.D. thesis, RWTH Aachen University (2018). https://doi.org/10.18154/RWTH-2018-229093

12. Kunz, E.: Introduction to Commutative Algebra and Algebraic Geometry. Birkhäuser, Boston (1985)

13. Llibre, J., Valls, C., Zhang, X.: Liouvillian integrability versus Darboux polynomials. Qual. Theory Dyn. Syst. 15, 503-515 (2016)

14. Perko, L.: Differential Equations and Dynamical Systems, 3rd edn. Springer, New York (2001)

15. Schilli, C., Zerz, E., Levandovskyy, V.: Controlled and conditioned invariant varieties for polynomial control systems. in: Proceedings of the 21st International Symposium on Mathematical Theory of Networks and Systems, Groningen (2014)

16. Schilli, C., Zerz, E., Levandovskyy, V.: Controlled and conditioned invariance for polynomial and rational feedback systems. In: Advances in Delays and Dynamics: Algebraic and Symbolic Computation Methods in Dynamical Systems, vol. 9. Springer (2020)

17. Schilli, C.: Controlled and conditioned invariant varieties for polynomial control systems. Ph.D. thesis, RWTH Aachen University, 2016. https://publications.rwth-aachen.de/record/670544

18. Shafarevich, I.R.: Basic Algebraic Geometry. Springer, Berlin (1977)

19. Yuno, T., Ohtsuka, T.: Lie derivative inclusion for a class of polynomial state feedback controls. Trans. Inst. Syst. Control Inf. Eng. 27(11), 423-433 (2014)

20. Yuno, T., Ohtsuka, T.: Lie derivative inclusion with polynomial output feedback. Trans. Inst. Syst. Control Inf. Eng. 28(1), 22-31 (2015)

21. Zerz, E., Walcher, S.: Controlled invariant hypersurfaces of polynomial control systems. Qual. Theory Dyn. Syst. 11, 145-158 (2012)

22. Zhang, X.: Integrability of Dynamical Systems: Algebra and Analysis. Developments in Mathematics, vol. 47. Springer, Singapore (2017)

Publisher's Note Springer Nature remains neutral with regard to jurisdictional claims in published maps and institutional affiliations. 